\title{
Genome-wide identification and expression analysis of the potato ZIP gene family under Zn-deficiency
}

\author{
X. B. LI ${ }^{1,2}$, H.C. SUO ${ }^{2}$ J.T. LIU ${ }^{2}$, L. WANG ${ }^{2}$, C.C. $\mathrm{LI}^{2}$, and W. LIU ${ }^{1 *}$ \\ College of Life Sciences, South China Agricultural University, Guangzhou 510642, P.R. China \\ Crops Research Institute, Guangdong Academy of Agricultural Sciences / Guangdong Province Key \\ Laboratory of Crop Genetic Improvement, Guangzhou, Guangdong 510640, P.R. China ${ }^{2}$
}

\begin{abstract}
Zinc deficiency is a worldwide problem for crops including potato (Solanum tuberosum L.), the fourth most important crop worldwide. The zinc/iron-regulated transporter-like protein (ZIP) transporter family is thought to play key roles in $\mathrm{Zn}$ uptake and transport. However, little is known about the potato ZIP family. In this study, 12 genes encoding members of the ZIP family were identified in the potato genome. The 12 StZIP genes were predicted to encode proteins of 220 407 amino acids harboring 5 - 9 putative transmembrane domains (TMDs), and 11 of these proteins had a variable region rich in histidine residues between TMDIII and TMDIV. A phylogenetic analysis divided the StZIPs into four groups on the basis of gene structure and conserved motifs. Furthermore, the StZIP expression profiles were determined under Zndeficiency in both high and low Zn-content genotypes. Four differentially expressed genes, StZIP6, -9, -11, and -12, were identified in tubers of the two genotypes under Zn-deficiency, and StZIP11 and StZIP12 may have a more prominent function in $\mathrm{Zn}$ uptake and accumulation in potato tubers owing to their higher expressions. Thus, the results provide useful information for further studying the functions of StZIP genes.
\end{abstract}

Additional key words: Solanum tuberosum, transmembrane domains, Zn uptake and transport.

\section{Introduction}

Zinc is a key element in a large number of transcriptional regulatory proteins. It serves as a cofactor for many metalloenzymes and plays important roles in plant growth regulation, enzyme activation, gene expression and regulation, protein synthesis, photosynthesis, and saccharide metabolism (Marschner 1995, Guerinot 2000, Figueiredo et al. 2012). However, Zn deficiency is a widespread problem among crops (Hambidge 2000, Cakmak 2008), and approximately $40 \%$ of the total land area of China is $\mathrm{Zn}$ deficient (Yang et al. 2007). Zn deficiency impairs Zn-dependent physiological functions, leading to abnormality in plant growth (Marschner 1995, Rengel and Graham 1995, Lombnaes and Singh 2003) and causing decreases in yield and nutritional quality (Marschner 1995, Yusuf et al. 2002, Sadeghzadeh 2013).

In plants, $\mathrm{Zn}$, as a divalent cation or in complex with organic ligands, is translocated to the root xylem through symplast and apoplast pathways, and transported further through both xylem and phloem tissues (Haslett et al. 2001, Kabata-Pendias and Pendias 2001, Broadley et al. 2007). In recent years, several types of transporters, including P-type ATPase, multi-drug and toxic compound extrusion transporters, oligopeptide transporters, and zinc/ironregulated transporter-like protein (ZIP) transporters, have been found to be involved in $\mathrm{Zn}$ uptake and translocation (Lochlainn et al. 2011, Hu et al. 2012, Pineau et al. 2012). The ZIP transporter family plays key roles in Zn uptake and translocation (Eide et al. 1996, Grotz et al. 1998, Pence et al. 2000, Wintz et al. 2003, Lin et al. 2009, Milner et al. 2013). Most ZIP proteins are predicted to have eight potential transmembrane domains (TMDs), and the amino and carboxy terminal ends of the protein are located on the outside surface of the plasma membrane (Guerinot 2000). There is a variable region between TMDs $\amalg$ and IV that is

Submitted 13 May 2020, last revision 6 August 2020, accepted 3 September 2020.

Abbreviations: GSDS - gene structure display server tool; PGSC - Potato Genome Sequencing Consortium; RH - Solanum tuberosum group Tuberosum RH89-039-16; DM - Solanum tuberosum group Phureja DM1-3 516 R44; TMD - transmembrane domain; ZIP - zinc/ iron-regulated transporter-like protein.

Acknowledgements: The authors thank Lesley Benyon, PhD, from Liwen Bianji, Edanz Group China (www.liwenbianji.cn/ac), for editing the English text of a draft of this manuscript. This work was supported by the National Natural Science Foundation of China's Youth Fund (31701486), Key-Area Research and Development Program of Guangdong Province (2020B020219002), Guangdong Provincial Science and Technology Program (2017B020232002), and The National Key Research and Development Program of China (2018YFD020080902). The first two authors contributed equally to this work.

* Corresponding author; e-mail: liuwei@scau.edu.cn 
predicted to reside in the cytoplasm; this region contains a potential metal-binding domain, rich in histidine residues, which is associated with transport (Guerinot 2000, Mäser et al. 2001). To date, ZIP family members have been identified in bacteria, archaea, and all types of eukaryotes such as animals, plants, protists, and fungi (Mäser et al. 2001). In Arabidopsis thaliana, 15 ZIP members have been found, including AtIRT1-3 and AtZIP1-12, which share sequence similarities of 38 - $85 \%$ (Mäser et al. 2001). A phylogenetic analysis using amino acid sequence divided these ZIP proteins into four groups, with one of the groups clearly being more distantly related (Mäser et al. 2001). Among them, AtIRT1-3, AtZIP1-3, -7, -11, and -12 were able to functionally complement a yeast $\mathrm{Zn}$ uptake-deficient mutant (Eide et al. 1996, Grotz et al. 1998, Guerinot 2000, Haslett et al. 2001, Vert et al. 2001, 2002, 2009, Lin et al. 2009, Milner et al. 2013). The expressions of some ZIPs, including AtZIP1, -5, -9, -12, and AtIRT3, increase under Zn-limiting conditions (Krämer et al. 2007). The overexpression of AtZIP1 increases short-term Zn uptake and seed Zn content in barley and cassava (Ramesh 2004, Gaitánsolís et al. 2015). In rice, 16 OsZIPs members have been identified by homology cloning or a real-time PCRbased strategy (Ramesh and Schachtman 2003, Ishimaru et al. 2005, Yang et al. 2009, Chandel et al. 2010). Under $\mathrm{Zn}$ deficiency, the expressions of OsZIP1, -3,-6, and -8, which complement the phenotype of a $\mathrm{Zn}$ uptake-deficient yeast mutant, are upregulated in both roots and shoots (Yang et al. 2009, Lee et al. 2010a,b, Kavitha et al. 2015). Additionally, the expressions of OsZIP2 and OsZIP7 are specifically upregulated in roots and shoots, respectively (Ramesh and Schachtman 2003, Ishimaru et al. 2005). OsZIP3, located in the node, is responsible for unloading $\mathrm{Zn}$ from the xylem of enlarged vascular bundles (Sasaki et al. 2015). The ectopic expression of OsZIP1 or OsZIP4 increases the $\mathrm{Zn}$ content in tobacco and finger millet seeds or sweet potato tuber, respectively (Ramegowda et al. 2013, Shin et al. 2016). The overexpression of OsZIP4 or OsZIP5 results in a disarranged $\mathrm{Zn}$ distribution in rice (Ishimaru et al. 2007, Lee et al. 2010a). Thus, ZIP proteins play key roles in plant responses to $\mathrm{Zn}$ deficiency. Over the past decade, the functions of ZIP members have been identified and investigated in many species, such as Glycine max (Moreau et al. 2002), Medicago sativa (López-Millán et al. 2004, Stephens et al. 2011), Hordeum vulgare (Tiong et al. 2014), Zea mays (Li et al. 2013), and Vitis vinifera (Gainza-Cortés et al. 2012). However, ZIPs have not been explored in potato, even though it is a globally important crop (White et al. 2017). To provide key information for the further identification and functional analysis of StZIP genes in potato, 12 members of the ZIP family were identified from the potato genome, and their phylogenetic relationships, transmembrane domains, conserved protein motifs, gene structures, chromosomal locations, and promoters were analyzed. In addition, the tuber $\mathrm{Zn}$ content of 76 potato genotypes and a cluster analysis were performed. The differences in the StZIP expression patterns in high and low $\mathrm{Zn}$-content genotypes in response to $\mathrm{Zn}$ deficiency were investigated.

\section{Materials and methods}

Identification of potato ZIP family members: Potato protein sequence data (DM_v3.4_pep_nonredundant) were downloaded from the Potato Genome Sequencing Consortium (PGSC, http://potato.plantbiology.msu.edu/ integrated -searches.shtml). The hidden Markov model $(H M M)$ was used to identify potato ZIP candidates, and the hidden Markov model profile of ZIP (PF02535) was downloaded from Pfam (http://pfam.xfam.org). A HMMER search was carried out using HMMER software with e-value $\leq 1 \mathrm{e}^{-1 \mathrm{e}}$. The outputs of putative protein sequences were analyzed using $C D D$ (https://www.ncbi. nlm.nih.gov/Structure/bwrpsb/bwrpsb.cgi), Pfam, and SMART (http://smart.embl-heidelberg.de/) to confirm the presence of conserved domains. All nonredundant and high-confidence genes were assigned as potato StZIPs. The gene locus, amino acid length, chromosomal location, and other information on each StZIP was acquired from $P G S C$. The exon/intron organization of StZIP genes were analyzed using the Gene Structure Display Server tool (GSDS, http://gsds.cbi.pku.edu.cn/). The TMDs of the ZIPs were predicted using CCTOP (http://cctop.enzim. ttk.mta.hu/?ref=labworm). Molecular mass and isoelectric point were calculated using the online program provided by the ExPASy website (http://web.expasy.org/protparam/).

Multiple sequence alignment and phylogenetic analysis: Multiple sequence alignment was performed using Clustal $X$ 2.0. The phylogeny of the ZIP proteins was constructed using MEGA v. 7.0 by the neighborjoining method with 1000 bootstrap replications. The phylogenetic tree was modified using the online software EvolView (https://evolgenius.info//evolview-v2/\#login). For sequence logo analysis, the variable region sequences from potato, tomato, pepper, Arabidopsis, and rice were aligned, and the multiple alignment result was submitted to an online tool, WEBLOG (http://weblogo.berkeley.edu/ logo.cgi).

Gene structure and conserved motif characterization of potato ZIPs: Conserved motif residues in potato ZIP sequences were determined using $M E M E$ suite with maximum motif number of 5 , and minimum and maximum motif widths of 6 and 50, respectively (http://meme-suite. org/tools/meme). The predicated motif sequences were annotated using CDD, SMART, and InterPro (http://www. ebi.ac.uk/interpro/). The exon-intron structures of the StZIP genes were identified using GSDS (http://gsds.cbi. pku.edu.cn/).

Chromosomal location: The chromosomal positions of StZIP genes were obtained using the potato genome browser at PGSC. The approximate locations of ZIP genes were mapped to potato chromosomes using Mapchart software. Gene duplication was confirmed on the basis of two criteria: 1 ) the length of the shorter aligned sequence covering $>70 \%$ of the longer sequence, and 2) the similarity between the two aligned sequences being $>70 \%$ (Gu et al. 2002, Yang et al. 2008). Two genes separated 
by five or fewer genes in a $100 \mathrm{~kb}$ chromosomal fragment were considered to be tandemly duplicated genes (Deng et al. 2014).

Promoter analysis: The $1500 \mathrm{bp}$ upstream sequences from the transcriptional start sites of StZIP genes were analyzed using PlantCARE (http://bioinformatics.psb. ugent.be/webtools/plantcare/html/), and the results were modified using GSDS.

Transcriptional expression pattern analysis of StZIP genes: The transcriptional expression patterns of StZIP genes in different tissues were determined using RNAseq data generated from $P G S C$. The read per kilobase of exon model per million reads mapped values of the ZIP genes were downloaded and $\log _{2}$-transformed, and the expression patterns were displayed using a heatmap generated by HemI 1.0 (Deng et al. 2014).

Plant materials and treatments: Healthy tubers of potato (Solanum tuberosum L.) genotypes L14148-5 and Minshu1 were cut into uniform size samples and sterilized with $3 \%(\mathrm{~m} / \mathrm{v})$ carbendazim. They were then planted in pots with moistened silica sand (one tuber per pot) and cultured in growth chambers at day/night temperatures of $25 / 20^{\circ} \mathrm{C}$, a 16-h photoperiod, an irradiance of $60 \mu \mathrm{mol} \mathrm{m} \mathrm{m}^{-2} \mathrm{~s}^{-1}$, and a relative humidity of $50 \%$. After the potato seedlings emerged, the mother tubers were removed. Seedlings were divided into two groups. The first group was watered with Hoagland's solution as described by Fong and Ulrich (1969) once a week, and the second group was watered with the Hoagland's solution minus $\mathrm{Zn}$ once a week, representing Zn-deficiency condition. The leaves and tubers were sampled at 60 and $90 \mathrm{~d}$ after treatment, respectively, from three individual plants growing under Zn-sufficient and Zn-deficient conditions. All the samples were immediately frozen in liquid nitrogen and stored at $-80{ }^{\circ} \mathrm{C}$ for further processing.

Determination of $\mathbf{Z n}$ content: Potato tubers were dried at $65^{\circ} \mathrm{C}$ for 1 week. The 25 -mg samples were wet-ashed with $5 \mathrm{~cm}^{3}$ of $\mathrm{HNO}_{3}$ and $1 \mathrm{~cm}^{3}$ of $\mathrm{HClO}_{4}$ at $150{ }^{\circ} \mathrm{C}$ for $5 \mathrm{~h}$. The $\mathrm{Zn}$ content was measured using a flame atomic absorption spectrophotometer (ZA3300, Hitachi, Tokyo, Japan) at a wavelength of $213.86 \mathrm{~nm}$.

Real-time PCR analysis was performed on a CFX96 realtime PCR detection system (Bio-Rad, Hercules, USA) using SYBR ${ }^{\circledR}$ Premix Ex TaqTM II (Tli RHaseH Plus) (TaKaRa, Tokyo, Japan). All the reactions were carried out in a $25 \mathrm{~mm}^{3}$ volume containing $1 \mathrm{~mm}^{3}$ of cDNA as the template. The two-step thermal cycling conditions consisted of $95{ }^{\circ} \mathrm{C}$ for $30 \mathrm{~s}$, followed by 39 cycles of $95^{\circ} \mathrm{C}$ for $5 \mathrm{~s}$, and $60^{\circ} \mathrm{C}$ for $30 \mathrm{~s}$. The $e f 1-\alpha$ was used as an internal control. Each PCR assay included three biological replicates and three technical replicates. PCR data were evaluated using the $2^{-\Delta \mathrm{Ct}}$ method. The specific primers used for each gene are listed in Table 2 Suppl.

\section{Results}

In total, 26 putative sequences were obtained by the HMMER search, and 12 sequences were identified as potato ZIP proteins after confirming the presence of characteristic conserved domains using CDD, Pfam, and SMART. The StZIPs were mainly characterized as having 2 - 4 exons encoding 220 - 407 aa, with molecular masses ranging from 23.67 to $43.41 \mathrm{kDa}$ and isoelectric points ranging from 5.4 to 9.03 . Detailed information on their characteristics is listed in Table 1. The ZIP genes were named StZIP1 to StZIP12 in accordance with their chromosomal locations.

The StZIP proteins were predicted to have five to 9 putative TMDs, and five StZIPs, StZIP5, -6, -8, -9, and -12 , were predicted to have eight putative TMDs (Table 1, Fig. 1 Suppl.). All the StZIP proteins, except for StZIP11, had a long loop region between TMDIII and TMDIV that is referred to as the variable region. The variable region contained a potential metal-binding domain that is rich

Table 1. Features of Solanum tuberosum zinc/iron-regulated transporter-like protein (StZIP) genes identified in potato. TMD transmembrane domain, $\mathrm{pI}$ - isoelectric point, $\mathrm{Mr}$ - molecular mass.

\begin{tabular}{llllllll}
\hline Gene name & Genomic ID & AA [aa] & No. of exons & No. of TMDs & pI & Mr [kDa] & Chromosome location \\
\hline StZIP1 & PGSC0003DMG400014936 & 348 & 3 & 9 & 6.50 & 37.239 & chr02: 15695173,15697155 \\
StZIP2 & PGSC0003DMG400010343 & 281 & 2 & 6 & 8.81 & 30.673 & chr02: 28265610,28267700 \\
StZIP3 & PGSC0003DMG400010344 & 331 & 3 & 9 & 8.48 & 35.691 & chr02: 28278147,28279390 \\
StZIP4 & PGSC0003DMG400021155 & 360 & 3 & 9 & 9.03 & 38.874 & chr02: 28347797,28350229 \\
StZIP5 & PGSC0003DMG400017732 & 354 & 3 & 8 & 8.16 & 38.436 & chr02: 37391695,37394478 \\
StZIP6 & PGSC0003DMG400027159 & 320 & 2 & 8 & 5.96 & 35.244 & chr05:48633733,48635684 \\
StZIP7 & PGSC0003DMG400002151 & 334 & 3 & 9 & 5.63 & 36.483 & chr06: 4560296,4563027 \\
StZIP8 & PGSC0003DMG400013109 & 340 & 3 & 8 & 6.14 & 36.434 & chr07: 41345919,41348870 \\
StZIP9 & PGSC0003DMG400013108 & 333 & 3 & 8 & 6.05 & 35.589 & chr07: 41354134,41357585 \\
StZIP10 & PGSC0003DMG400011747 & 220 & 3 & 5 & 7.07 & 23.667 & chr07: 41452935,41456033 \\
StZIP11 & PGSC0003DMG400022179 & 349 & 3 & 9 & 5.40 & 37.528 & chr07: 55406651,55409430 \\
StZIP12 & PGSC0003DMG400029937 & 407 & 4 & 8 & 6.14 & 43.405 & chr08: 35990888,35994505 \\
\hline
\end{tabular}




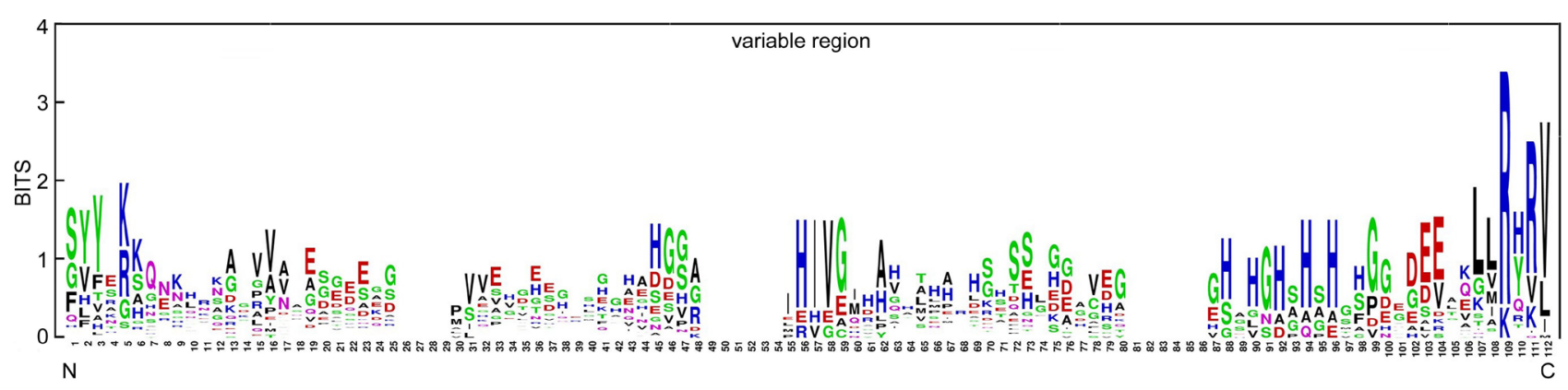

Fig. 1. Sequence logos showing variable regions of 12 zinc/iron-regulated transporter-like proteins (ZIPs) from potato, 10 ZIPs from tomato, 4 ZIPs from pepper, 15 ZIPs from Arabidopsis, and 10 ZIPs from rice.

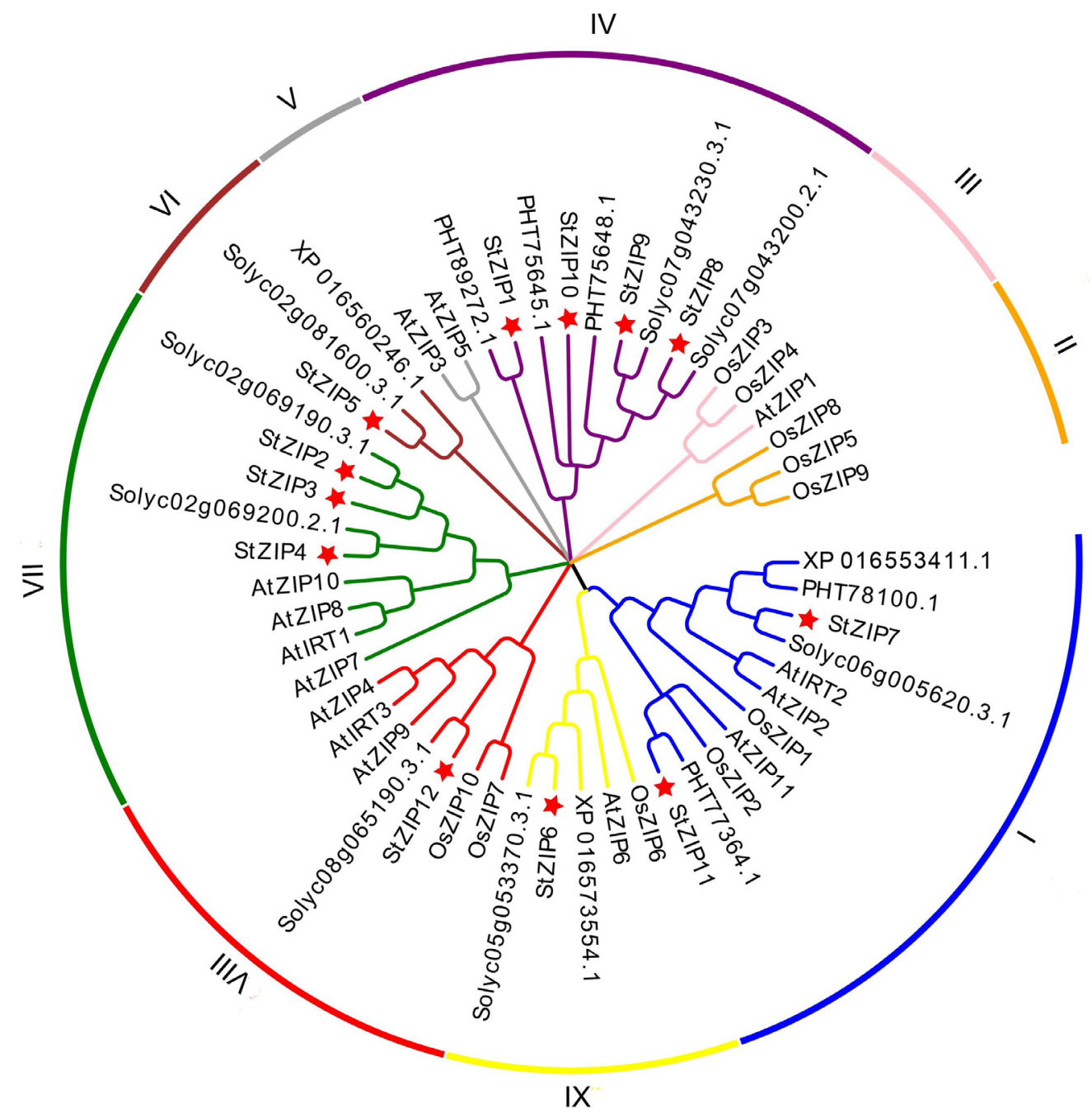

Fig. 2. A phylogenetic tree of zinc/iron-regulated transporter-like proteins (ZIPs) from Arabidopsis, rice, tomato, pepper, and potato constructed using the MEGA7.0 software by the neighbor-joining method with 1000 bootstrap replications. The nine subfamilies are distinguished by different colors.

in histidine. For example, in StZIP12, this sequence is HAHAAHHRHSHSHEH, and in StZIP8, this sequence is IHTHSHAHAH, suggesting a role in metal transport or regulation (Fig. 1, Fig. 1 Suppl.).

An unrooted phylogenetic tree was constructed using full-length amino acid sequences, with 10 sequences from rice, 14 sequences from Arabidopsis, 11 sequences from tomato, 5 sequences from pepper, and 12 sequences from potato. In total, 52 sequences were classed into 9 subgroups. The 12 potato ZIPs had members in 6 subgroups, except subgroups II, III, and V. Within the same subgroups, potato ZIP proteins were more closely 


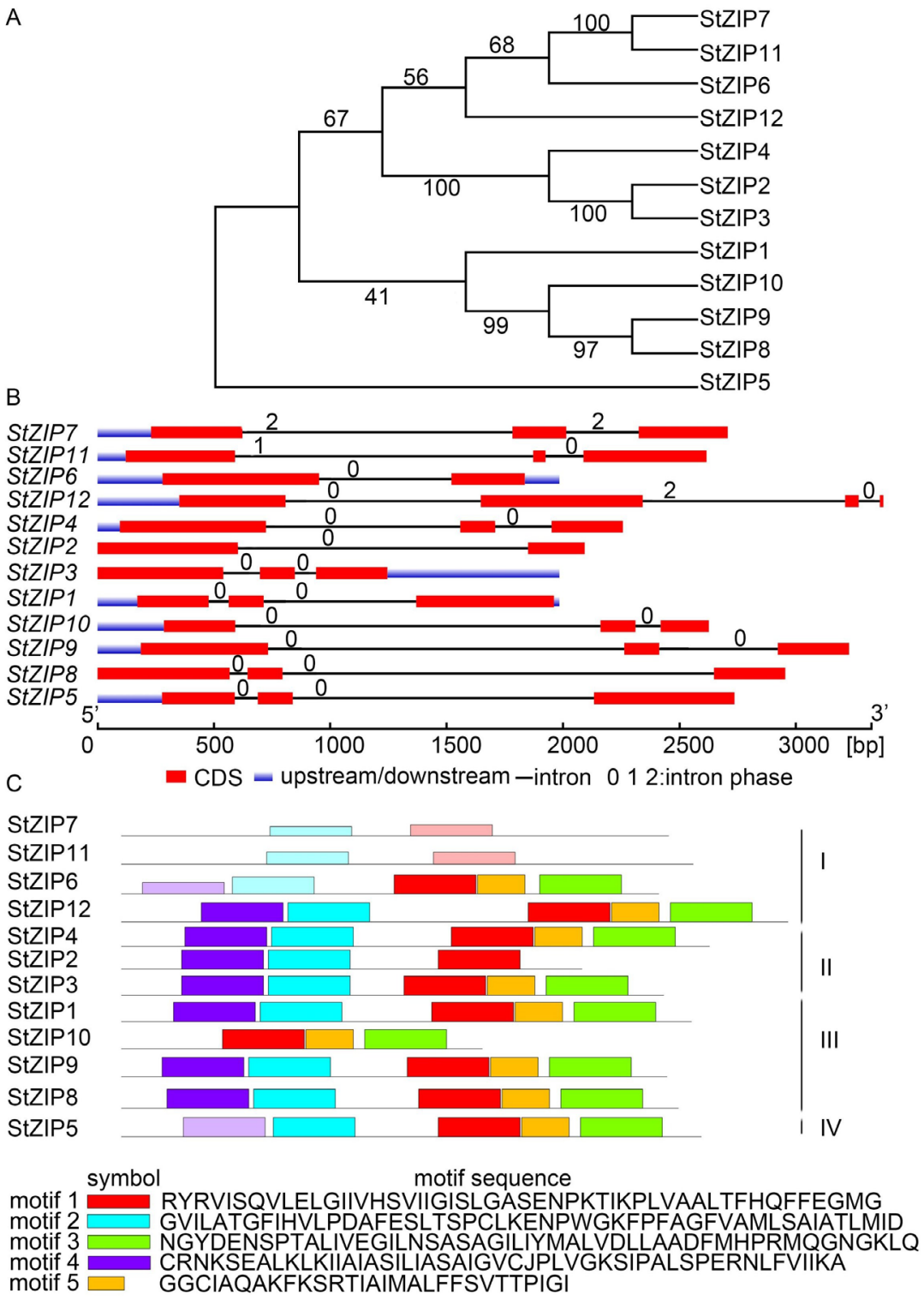

Fig. 3. The evolutionary relationships, gene structures, and functional motifs of the Solanum tuberosum zinc/iron-regulated transporterlike proteins $(S t Z I P S)$. A - a phylogenetic tree constructed using the MEGA7.0 software by the neighbor-joining method with 1000 bootstrap replications. $B$ - exon/intron distributions of corresponding StZIP genes detected by comparing the predicted coding sequences with their corresponding genomic sequences using gene structure display server tool online. $C$ - motif composition of each StZIP protein. Motifs 1 - 5 are displayed as differently colored boxes with the corresponding sequence information for each motif. The lighter colors represent the lower similarity with the motif sequences.

related to tomato or pepper ZIP proteins than rice and Arabidopsis ZIP proteins. Subgroup I was the largest, with 11 members, while subgroup $\mathrm{V}$ was the smallest, containing only 2 members. Most ZIP proteins in the same subgroup shared similar exon-intron structures. For instance, subgroup IX had only one intron, subgroups VI and V had two introns, and subgroup VIII had three introns except for AtZIP9 (Table 1 Suppl.). In addition, StZIP2, -3 , and -4 located on chromosome 2, and StZIP8-10 located on chromosome 7 clustered in subgroups VII and IV, respectively, suggesting that they might represent tandemly duplicated genes (Fig. 2).
Structures and phases of introns/exons were determined by the alignment of genomic DNA with the full-length cDNA of the StZIPs (Fig. 3B). The number of introns varied from one to three. Nine StZIP members (75\%) had two introns, two members $(16.7 \%)$ had only one intron, while only one member had three introns. Three intron phases, termed zero, one, and two, were generally present when splicing occurred after the third, first, and second nucleotides, respectively. Seven members, StZIP1, StZIP3StZIP5, and StZIP8-StZIP10, had intron phase $(0,0)$, StZIP7 had intron phase $(2,2)$, and StZIP11 had intron phase $(1,0)$. StZIP2 and StZIP6 had only one intron with 


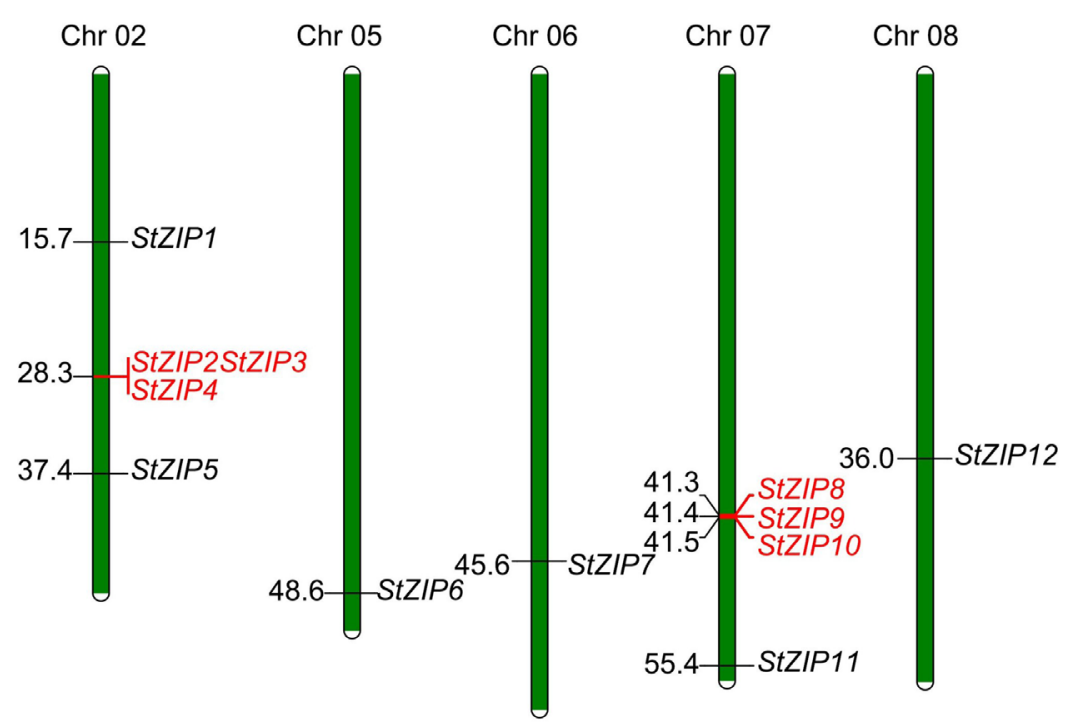

Fig. 4. Chromosomal locations of Solanum tuberosum zinc/iron-regulated transporter-like protein (StZIP) genes mapped using information available from the potato genome database. Tandemly duplicated genes are marked in red, the scale is in megabases.

intron phase (0) and StZIP12 had the most introns with intron phase $(0,2,0)$ (Fig. $3 B)$.

The conserved motifs of the StZIPs were analyzed using $M E M E$ software. In total, five conserved motifs, having lengths of 29 to 50 aa, and one copy of all motifs was found in each StZIP protein. The motif sequences were annotated according to CDD, SMART, and Pfam, and motifs 1, 3, and 4 were predicted to form a transmembrane helical region. Each member contained different numbers of conserved motifs, ranging from two to five. Motif 1 was found in all StZIP proteins, and five conserved motifs were found in the majority of proteins $(66.7 \%)$. Three motifs were found in other members, such as StZIP10 and StZIP2, while only two motifs were found in StZIP7 and StZIP11. The different conserved motif profiles may indicate functional diversity. In addition, the StZIPs were subdivided into four groups on the basis of the phylogenetic analysis, and proteins in the same group of the phylogenetic tree shared similar motif structures (Fig. 3C).

The StZIP genes were unevenly distributed on five potato chromosomes. Chromosome 2 had most StZIP genes - five, chromosome 7 contained four members, while only one gene was found on the other chromosomes. The majority of StZIP genes were located on the proximate or distal ends of the chromosomes. On the basis of the two criteria, six (50\%) StZIP genes were confirmed to be tandemly duplicated genes. StZIP2, -3 , and -4 formed a duplicated gene cluster, and StZIP8, -9 , and -10 formed another duplicated gene cluster (Fig. 4).

To understand the transcriptional regulatory mechanisms of the StZIP gene family, the promoter region's cis-regulatory elements located in the sequence 1500 -bp upstream of the transcriptional start sites were analyzed using PlantCARE. Many cis-regulatory elements were identified in this region. Most cis-regulatory elements were associated with important physiological processes such as in defense and responses to stress, radiation, and hormones. For example, the ATCT motif, Box4,
GATA motif, and GT1 motif are involved in radiation responsiveness; myoblastosis and TC-rich motifs are involved in defense and stress responsiveness; ABRE is involved in ABA responsiveness; and the TGA element is involved in auxin responsiveness. In addition, other uncommonly distributed cis-regulatory elements that are functionally associated with transcription modulation, endosperm and meristem expression, leaf differentiation and development, and circadian control were found. Thus, StZIP genes may be regulated by various environmental and developmental changes. Duplicated gene pairs did not share similar cis-element distributions. These observations suggest the involvement of duplication events in conferring neofunctionalization to StZIP genes during their divergence and evolvement (Fig. 5).

To analyze the expression patterns of StZIP genes in various tissue types and organs, the read per kilobase of exon model per million mapped reads values of two potato species, Solanum tuberosum group Tuberosum RH89039-16 (RH) and doubled monoploid Solanum tuberosum group Phureja DM1-3 516 R44 (DM), were downloaded from PGSC and a heatmap was generated. StZIP5, -9, -11, and-12 had relatively high expression in all tissue types for both RH and DM. StZIP1 was highly expressed in flowers, leaves, and stem, but not tubers, in both RH and DM. The expressions of StZIP2, -3, and -4 were mainly detected in flowers, suggesting that they may be related to flower development. In addition, StZIP 2 showed a relatively high expression in the root of DM. StZIP6 showed a moderate expression in the stem of $\mathrm{RH}$ and in the flower and roots of DM. StZIP7 was highly expressed in the root and stolons of DM. StZIP10 exhibited moderate expression in RH leaves and stem. StZIP 8 expression was nearly undetectable in all the tissue types except for RH leaves. Thus, StZIP members exhibited very different expression profiles in different organs for the two potato species. StZIP1,-4,-5,-9,-10,-11, and -12 have similar expression patterns in these two potato genotypes, while StZIP2,-3,-6,-7 and -8 showed 
AABRE $\square$ AT-rich $D$ ATCT-motif $D$ Box4 D GATA-motif GT1-motif $\square$ MYB $\square$ TATC-box $\square$ TC-rich TGA-element

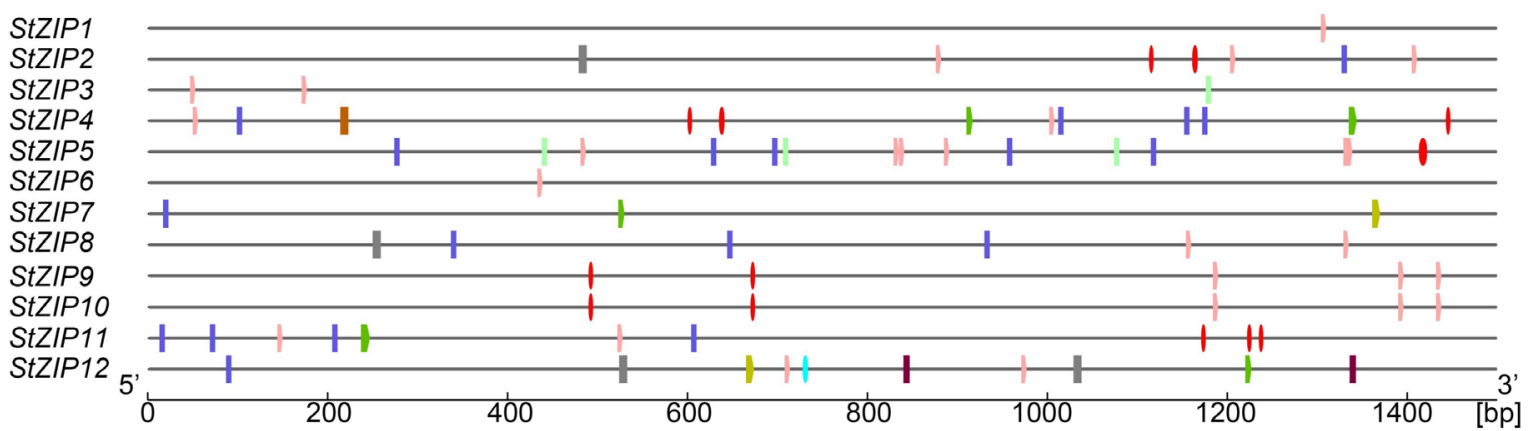

Fig. 5. Promoter analysis of Solanum tuberosum zinc/iron-regulated transporter-like protein (StZIP) genes. The legend shows different stress-response elements located in the $1.5 \mathrm{~kb}$ 5' upstream region of each StZIP gene.

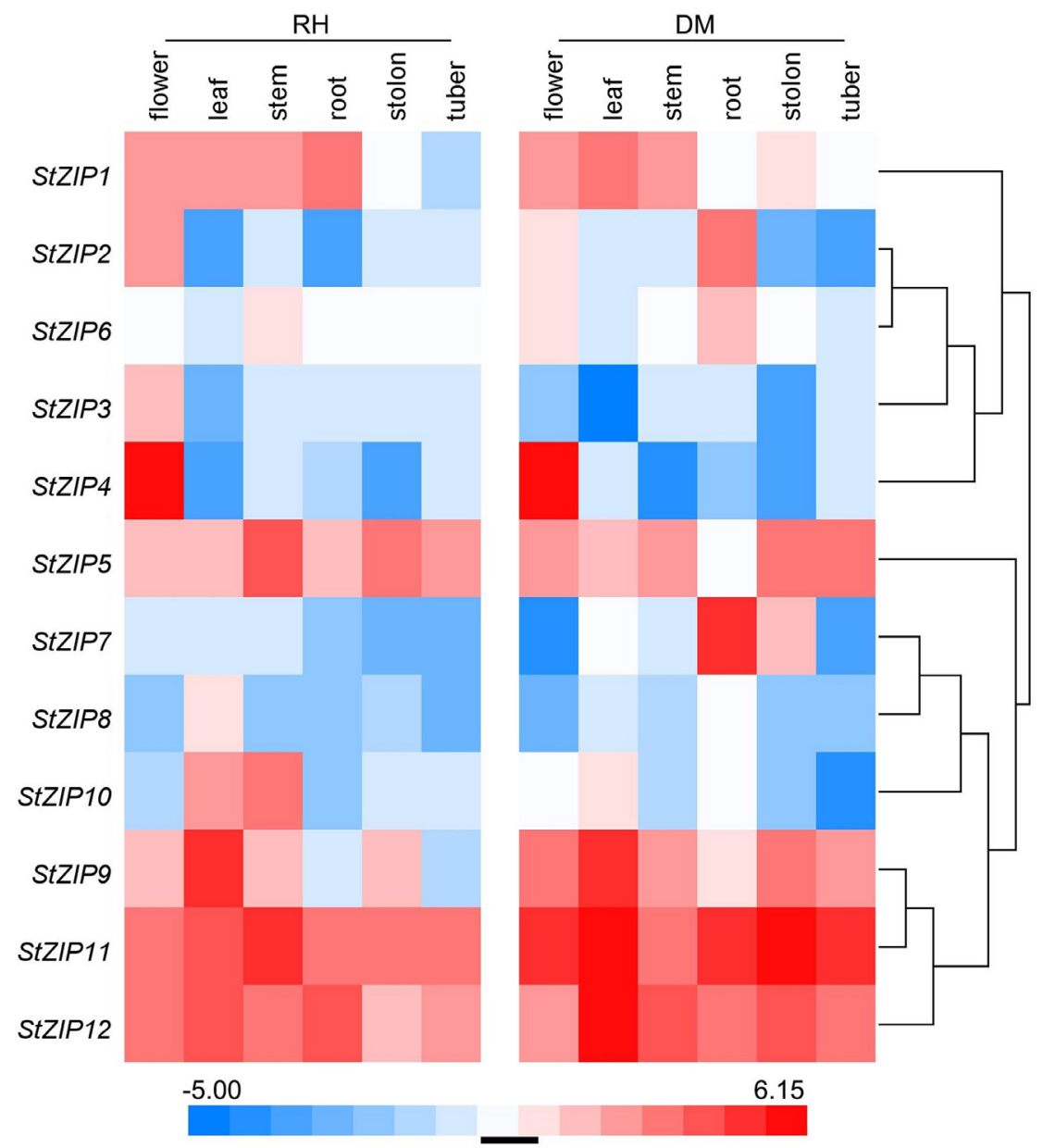

Fig. 6. Expression profiles of Solanum tuberosum zinc/iron-regulated transporter-like protein (StZIP) genes in different potato organs and tissue types. A heatmap generated by HemI 1.0 using reads per kilobase of exon model per million reads mapped values of all the ZIP genes that were downloaded from the Potato Genome Sequencing Consortium and $\log _{2}$-transformed. DM - doubled monoploid Solanum tuberosum goup Phureja DM1-3, RH - Solanum tuberosum group Tuberosum RH89-039-16.

significantly different expressions (Fig. 6).

The expression patterns of StZIPS in potato were further studied to understand their roles in $\mathrm{Zn}$ uptake and translocating under Zn-deficiency conditions. First, the tuber $\mathrm{Zn}$ content of 76 potato genotypes were evaluated, and the genotypes clustered into four subgroups on the basis of their Zn content. Subgroup I, containing eight potato genotypes, had the highest $\mathrm{Zn}$ content ranging from 31.44 to $46.90 \mathrm{mg} \mathrm{kg}^{-1}$ (f.m.). Subgroup II, containing 13 genotypes, was a middle-to-high $\mathrm{Zn}$ content group with $\mathrm{Zn}$ content ranging from 25.07 to $29.9 \mathrm{mg} \mathrm{kg}^{-1}$ (f.m.). Subgroup III, containing 18 genotypes, was a middle 
Table 2. Tuber $\mathrm{Zn}$ content in two potato genotypes under normal $(\mathrm{CK})$ and $\mathrm{Zn}$-deficiency conditions. ** - significant differences at $P \leq 0.01$.

\begin{tabular}{lll}
\hline & CK & Zn Deficiency \\
\hline L14148-5 & $39.50 \pm 1.64$ & $37.62 \pm 2.69^{* *}$ \\
Minshu1 & $17.00 \pm 0.43$ & $15.44 \pm 0.45^{* *}$ \\
\hline
\end{tabular}

Zn content group with $\mathrm{Zn}$ content ranging from 20.48 to $23.76 \mathrm{mg}^{-1}$ (f.m.), and subgroup VI, containing the most genotypes, was the lowest $\mathrm{Zn}$ content ranging from 12.69 to $20.08 \mathrm{mg} \mathrm{kg}^{-1}$ (f.m.) (Fig. 1 Suppl.).

Two genotypes, L14148-5 in subgroup I and Minshu1 in subgroup VI, were selected to explore the expression patterns of StZIP members. Additionally, we detected their tuber $\mathrm{Zn}$ content under Zn-deficiency. Even under these conditions, L14148-5 maintained a twofold greater content of $\mathrm{Zn}$ in tubers than Minshu1 (Table 2).

The expressions of three genes, StZIP5, -11, and -12, were higher in leaves and tubers compared with the other genes. In leaves, the expression of StZIP1 was induced, while the expressions of StZIP5, -7, -8, -10, and -11 were repressed under Zn-deficiency in both genotypes. The expressions of some other genes presented genotype-based differences, such as StZIP3 and StZIP9, which were reduced in L14148-5 but induced in Minshul under Zn deficiency. StZIP4 showed an opposite expression pattern in response to $\mathrm{Zn}$ deficiency, being induced in L14148-5 and repressed in Minshu1. In addition, some genes showed expression changes in only one genotype. For example, under Zn- deficiency, the expression of StZIP2 was repressed only in L14148-5, the expression of StZIP12 was induced only in Minshu1, and the expression of StZIP6 was repressed only in Minshu1. With regard to tubers, the expressions of StZIP1, -2, -3, -4, -7, and -8 were induced, while StZIP5 and StZIP10 were reduced under Zn-deficiency in both genotypes. The expressions of four other genes, StZIP6, $-9,-11$, and -12 , showed genotypic differences in response to $\mathrm{Zn}$ deficiency, and were increased in L14148-5 but decreased in Minshu1 (Fig. 7).

\section{Discussion}

In this study, we identified 12 ZIP members in potato (Table 1). There are 16 identified ZIP members in Oryza sativa, 15 in Arabidopsis thaliana, and 12 in Citrus reticulate, which indicate that the ZIP family is relatively small. The protein lengths of the potato, Arabidopsis, and orange ZIP members are 220 - 407 aa, 326 - 425 aa, and 334 - 419 aa (Fu et al. 2017). Potato ZIP members were predicted to have 6 - 9 TMDs, with the majority having 8 TMDs (Table 1, Fig. 1 Suppl.). Between TMDIII and TMDIV in all the StZIPs, except StZIP11 (Fig. 1 Suppl.), there is a variable region that is rich in histidine that thought to be a cytoplasmic metal ion-binding site (Guerinot 2000). The StZIP11 similar to AtZIP7, AtZIP11, MtZIP1, MtZIP7, and PtZIP2 (López-Millán et al. 2004, Fu et al. 2017), was found that the histidine-enriched region can be found at other different locations (Eng et al. 1998). StZIP12 has the greatest number of histidine residues in the variable region, which implies that it has a strong ability to bind

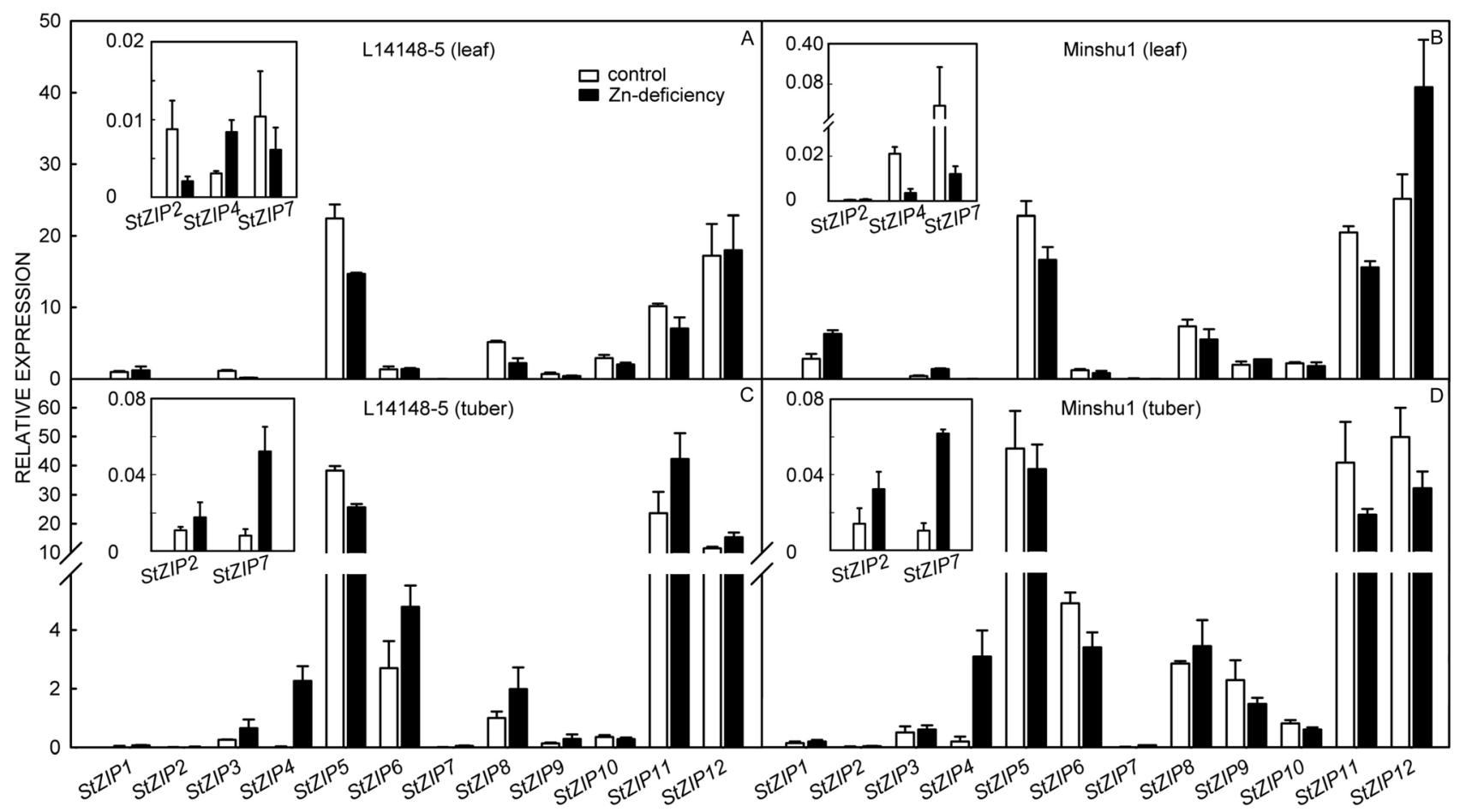

Fig. 7. Relative expressions of Solanum tuberosum zinc/iron-regulated transporter-like protein (StZIP) members in tubers and leaves of two potato genotypes in response to $\mathrm{Zn}$ deficiency. Values are means $\pm \mathrm{SEs}, n=5$. 
metal ions (Fig. 1). The 12 identified StZIP genes clustered into 4 subgroups, similar to the ZIP genes of Arabidopsis (Mäser et al. 2001). StZIP2, -3, and -4 are duplicated genes that cluster into the same phylogenetic subgroup and share a similar gene structure (Figs. 2 - 4). They were found to be highly expressed in flower organs, suggesting that they function in flower development (Fig. 6). Realtime PCR results showed that the expressions of StZIP2, $-3,-4$ were induced in tubers by the $\mathrm{Zn}$ deficiency, but their expressions in leaves showed genotype-based differences. StZIP3 expression was decreased in L141485, but increased in Minshu1. StZIP4 showed the opposite expression pattern to StZIP3, being induced in L14148-5 and repressed in Minshu1. The expression of StZIP2 was repressed only in the high $\mathrm{Zn}$ content genotype L14148-5 (Fig. 7). There was another cluster of duplicated genes, StZIP8, -9, and -10 (Fig. 4). StZIP8 was weakly expressed in leaves and roots, and StZIP9 had a relatively high expression in many tissues. The expression of StZIP10 was observed in leaves, petioles, and stems (Fig. 6). In leaves, StZIP8 and StZIP10 were repressed under Zn-deficiency in both genotypes, While StZIP9 was decreased in L14148-5, but increased in Minshu1. In the tubers of both genotypes under Zn-deficiency condition, StZIP8 was induced, while StZIP10 was repressed. StZIP9 expression also showed genotype-based differences, being increased in L14148-5, but decreased in Minshu1, in response to the $\mathrm{Zn}$-deficiency (Fig. 7). The functional diversity of the duplicated genes suggests that they may have evolved separate functions. Similar results were found in the research of duplicated genes in Poncirus trifoliata L. Three pairs of PtZIPs are both phylogenetically and physically close; however, there are no obvious similarities in their expression patterns and yeast complementation ( $\mathrm{Fu}$ et al. 2017). In other plants, like Arabidopsis and rice, the expression of some ZIPs are induced by Zn deficiency. AtZIP1, -5, -9, and -12, as well as AtIRT3, increase under Zn-limiting conditions (Krämer et al. 2007), while OsZIP7 is only upregulated in shoots (Ramesh and Schachtman 2003, Ishimaru et al. 2005). OsZIP1 - OsZIP6 and OsZIP8 are upregulated in roots and shoots under $\mathrm{Zn}$-deficiency and complement a $\mathrm{Zn}$ uptake-deficient yeast mutant (Ramesh and Schachtman 2003, Ishimaru et al. 2005, Yang et al. 2009, Lee et al. 2010a,b, Kavitha et al. 2015). In our research, we also found that some StZIPs were induced under Zn-deficiency, with StZIP1 induced in leaves and tubers, and StZIP2StZIP4, StZIP7, and StZIP8 induced in the tubers of both genotypes. We also found that the expression of some genes showed genotype-based differences in response to Zn-deficiency. StZIP3 and StZIP9 were only induced in the leaves of Minshu1, while StZIP6 and StZIP4 were only induced in the leaves of L1418-5. Additionally, StZIP9, -11 , and -12 were increased in tubers of L14148-5 but decreased in tubers of Minshul (Fig. 7). Compared with normal growth condition, four genes, StZIP6, -9, -11, and -12 , were differentially expressed in potato tubers among high and low $\mathrm{Zn}$ content genotypes under $\mathrm{Zn}$ deficiency (Fig. 7). The expressions of these four genes increased in L14148-4 and decreased in Minshu-1. In leaves, their expressions were unchanged or decreased under $\mathrm{Zn}$ deficiency compared with under normal growth condition in both genotypes (Fig. 7). The $\mathrm{Zn}$ content in the tubers of L14148-4 was more than twofold greater than in tubers of Minshu-1 under normal growth condition, and the former maintained the higher $\mathrm{Zn}$ content even under Zn-deficiency (Table 2). Thus, this indicates that the four differentially expressed genes, StZIP6, -9, -11, and -12, may have pivotal roles in $\mathrm{Zn}$ absorption and accumulation in potato tubers. Furthermore, StZIP6 and StZIP9 showed weak expression, while StZIP11 and StZIP12 showed much higher expressions in the leaves and tubers of both genotypes. The expression of StZIP11 was more than fivefold greater than those of StZIP6 and StZIP9, and the expression of StZIP12 was more than five- and 12-fold greater than those of StZIP6 and StZIP9, respectively (Fig. 7). StZIP11 and StZIP12 also had relatively high expressions in all the tissue types in RH and DM (Fig. 6), which suggests that StZIP11 and StZIP12 have prominent functions in the $\mathrm{Zn}$ uptake and accumulation in potato tubers.

\section{Conclusions}

Twelve ZIP genes were identified and clustered using the potato genome for the first time. We systematically identified ZIP family members from the potato genome, and analyzed their phylogenetic relationships, conserved protein motifs, and chromosomal locations, and we investigated the expression patterns of StZIPs in high and low Zn-content potato genotypes under the Zn deficiency. The results indicated that $S t Z I P$ genes play important roles in $\mathrm{Zn}$ uptake and translocation. In future studies, we will further investigate the functions of StZIPs in response to $\mathrm{Zn}$ deficiency in potato in more detail.

\section{References}

Broadley, M.R., White, P.J., Hammond, J.P., Zelko, I., Lux, A. Zinc in plants. - New. Phytol. 173: 677-702, 2007.

Clijsters, H., Van, A.F.: Inhibition of photosynthesis by heavy metals.- Photosynth. Res. 7: 31-40, 1985.

Cakmak, I.: Enrichment of cereal grains with zinc: agronomic or genetic biofortification? - Plant Soil. 302: 1-17, 2008.

Chandel, G., Banerjee, S., Vasconcelos, M.: Characterization of the root transcriptome for iron and zinc homeostasis-related genes in indica rice (Oryza sativa L.). - J. Plant Biochem. Biotechnol. 19: 145-152, 2010.

Deng, W., Wang, Y., Liu, Z., Cheng, H., Xue, Y.: HemI: a toolkit for illustrating heatmaps. - PLoS ONE 9: e111988, 2014.

Eide, D., Broderius, M., Fett, J., Guerinot, M.L.: A novel iron-regulated metal transporter from plants identified by functional expression in yeast. - Proc. nat. Acad. Sci. USA 93: 5624-5628, 1996.

Eng, B.H., Guerinot, H.L., Eide, D., Saier, M.H.I.: Sequence analyses and phylogenetic characterization of the ZIP family of metal ion transport proteins. - J. Membr. Biol. 166: 1-7, 1998.

Figueiredo, D.D., Barros, P.M., Cordeiro, A.M., Serra, T.S., Lourenco, T., Chande, R.S., Oliveira, M.M., Saibo, N.J.: Seven zinc-finger transcription factors are novel regulators 
of the stress responsive gene OsDREB1B. - J. exp. Bot. 63: 3643-3656, 2012.

Fong, K.H., Ulrich, A.: Growing potato plants by the water culture technique. - Amer. J. Potato Res. 46: 269-272, 1969.

Fu, X.Z., Zhou, X., Xing, F.: Genome-wide identification, cloning and functional analysis of the zinc/iron-regulated transporterlike protein (ZIP) gene family in trifoliate orange (Poncirus trifoliata L. Raf.). - Front. Plant Sci. 8: 588, 2017.

Gainza-Cortés, F., Pérez-Dïaz, R., Pérez-Castro, R.: Characterization of a putative grapevine $\mathrm{Zn}$ transporter, VvZIP3, suggests its involvement in early reproductive development in Vitis vinifera. - BMC Plant Biol. 12: 111, 2012.

Gaitánsolís, E., Taylor, N.J., Siritunga, D., Stevens, W., Schachtman, D.P.: Overexpression of the transporters AtZIP1 and AtMTP1 in cassava changes zinc accumulation and partitioning. - Front. Plant Sci. 6: 492, 2015.

Grotz, N., Fox, T., Connolly, E.: Identification of a family of zinc transporter genes from Arabidopsis that respond to zinc deficiency. - Proc. nat. Acad. Sci. USA 95: 7220-7224, 1998.

Gu, Z., Cavalcanti, A., Chen, F.C., Bouman, P., Li, W.H.: Extent of gene duplication in the genomes of Drosophila, nematode, and yeast. - Mol. Biol. Evol. 19: 256-262, 2002.

Guerinot, M.L.: The ZIP family of metal transporters. - Biochim. biophys. Acta 1465: 190-198, 2000.

Hambidge, M.: Human zinc deficiency. - J. Nutr. 130: 1344S-1349S, 2000.

Haslett, B.S., Reid, R.J., Rengel, Z.: Zinc mobility in wheat: uptake and distribution of zinc applied to leaves or roots. Ann. Bot. 87: 379-386, 2001.

Hu, Y.T., Ming, F., Chen, W.W., Yan, J.Y., Xu, Z.Y., Li, G.X., Xu, C.Y., Yang, J.L., Zheng, S.J.: TcOPT3, a member of oligopeptide transporters from the hyperaccumulator Thlaspi caerulescens, is a novel $\mathrm{Fe} / \mathrm{Zn} / \mathrm{Cd} / \mathrm{Cu}$ transporter. - PloS ONE 7: e38535, 2012.

Ishimaru, Y., Masuda, H., Suzuki, M., Bashr, K., Takahashi, M., Makanishi, H., Mori, S., Nishizawa, N.K.: Overexpression of the OsZIP4 zinc transporter confers disarrangement of zinc distribution in rice plants. - J. exp. Bot. 58: 2909-2915, 2007.

Ishimaru, Y., Suzuki, M., Kobayashi, T.: OsZIP4, a novel zincregulated zinc transporter in rice. - J. exp. Bot. 56: 3207-3214, 2005.

Kabata-Pendias, A., Pendias, H.: Trace Elements in Soils and Plants. - CRC Press, Boca Raton - London - New York 2001.

Kavitha, P.G., Sam, K., Mathew, M.K.: Functional characterization of a transition metal ion transporter, OsZIP6 from rice (Oryza sativa L.). - Plant Physiol. Biochem. 97: 165-174, 2015.

Krämer, U., Talke, I.N., Hanikenne, M.: Transition metal transport. - FEFS Lett. 581: 2263-2272, 2007.

Lee, S., Jeong, H.J., Sun, A.K.: OsZIP5 is a plasma membrane zinc transporter in rice. - Plant mol. Biol. 73: 507-517, 2010a.

Lee, S., Kim, S.A., Lee, J.: Zinc deficiency-inducible OsZIP8 encodes a plasma membrane-localized zinc transporter in rice. - Mol. Cells 29: 551-558, 2010 b.

Li, S., Zhou, X., Huang, Y.: Identification and characterization of the zinc-regulated transporters, iron-regulated transporterlike protein (ZIP) gene family in maize. - BMC Plant Biol. 13: $114,2013$.

Lin, Y.F., Liang, H.M., Yang, S.Y.: Arabidopsis IRT3 is a zinc-regulated and plasma membrane localized zinc/iron transporter. - New Phytol. 182: 392-404, 2009.

Lochlainn, S., Bowen, H.C., Fray, R.G., Hammond, J.P., King, G.J., White, P.J., Graham, N.S., Broadley, M.R.: Tandem quadruplication of HMA4 in the zinc $(\mathrm{Zn})$ and cadmium $(\mathrm{Cd})$ hyperaccumulator Noccaea caerulescens. - PLoS ONE 6: e17814, 2011.
Lombnaes, P., Singh, B.R.: Varietal tolerance to zinc deficiency in wheat and barley grown in chelator buffered nutrient solution and its effect on uptake of $\mathrm{Cu}, \mathrm{Fe}$, and Mn. - J. Plant Nutr. Soil Sci. 166: 76-83, 2003.

López-Millán, A.F., Ellis, D.R., Grusak, M.A.: Identification and characterization of several new members of the ZIP family of metal ion transporters in Medicago truncatula. - Plant mol. Biol. 54: 583-596, 2004.

Marschner, H.: Mineral Nutrition of Higher Plants. $2^{\text {nd }}$ Ed. Academic press, London 1995.

Mäser, P., Thomine, S., Schroeder, J.I.: Phylogenetic relationships within cation transporter families of Arabidopsis. - Plant Physiol. 126: 1646-1667, 2001.

Milner, M.J., Seamon, J., Craft, E.: Transport properties of members of the ZIP family in plants and their role in $\mathrm{Zn}$ and Mn homeostasis. - J. exp. Bot. 64: 369-381, 2013.

Moreau, S., Thomson, R.M., Kaiser, B.N.: GmZIP1 encodes a symbiosis-specific zinc transporter in soybean. - J. biol. Chem. 277: 4738-4746, 2002.

Pence, N.S., Larsen, P.B., Ebbs, S.D.: The molecular physiology of heavy metal transport in the $\mathrm{Zn} / \mathrm{Cd}$ hyperaccumulator Thlaspi caerulescens. - Proc. nat. Acad. Sci. USA 97: 49564960, 2000.

Pineau, C., Loubet, S., Lefoulon, C., Chalies, C., Fizames, C., Lacombe, B., Ferrand, M., Loudet, O., Berthomieu, P., Richard, O.: Natural variation at the FRD3 MATE transporter locus reveals cross-talk between $\mathrm{Fe}$ homeostasis and $\mathrm{Zn}$ tolerance in Arabidopsis thaliana. - PLoS Genet. 8: e1003120, 2012.

Ramegowda, Y., Ramegowda, V., Pavithra, J., Geetha, G., Rajashekar-Reddy, H., Udayakumar, M., Shanka, A.G.: Expression of a rice $\mathrm{Zn}$ transporter, OsZIP1, increases $\mathrm{Zn}$ concentration in tobacco and finger millet transgenic plants. - Plant Biol. Rep. 7: 309-319, 2013.

Ramesh, S.A., Choimes, S., Schachtman, D.P.: Over-expression of an Arabidopsis zinc transporter in Hordeum vulgare increases short-term zinc uptake after zinc deprivation and seed zinc content. - Plant mol. Biol. 54: 373-385, 2004.

Ramesh, S.A., Schachtman, D.P.: Differential metal selectivity and gene expression of two zinc transporters from rice. - Plant Physiol. 133: 126-134, 2003.

Rengel, Z., Graham, R.D.: Wheat genotypes differ in Zn efficiency when grown in chelate-buffered nutrient solution: II. Nutrient uptake. - Plant Soil 176: 317-324, 1995.

Sadeghzadeh, B.A.: Review of zinc nutrition and plant breeding. - J. Soil Sci. Plant Nutr. 13: 905-927, 2013.

Sasaki, A., Yamaji, N., Mitaniueno, N.: A node-localized transporter OsZIP3 is responsible for the preferential distribution of $\mathrm{Zn}$ to developing tissues in rice. - Plant J. cell. mol. Biol. 84: 374-384, 2015.

Shin, Y., Takahashi, R., Nakanishi, H.: Sweet potato expressing the rice $\mathrm{Zn}$ transporter OsZIP4 exhibits high $\mathrm{Zn}$ content in the tuber. - Plant Biotechnol. 33: 99-104, 2016.

Stephens, B.W., Cook, D.R., Grusak, M.A.: Characterization of zinc transport by divalent metal transporters of the ZIP family from the model legume Medicago truncatula. - Biometals 24: 51-58, 2011.

Tiong, J.L., McDonald, G.K., Genc, Y., Pedas, P., Hayes, J.E., Toubia, J., Langridge, P., Huang, C.Y.: HvZIP7 mediates zinc accumulation in barley (Hordeum vulgare) at moderately high zinc supply. - New Phytol. 201: 131-143, 2014.

Velu, G., Ortiz-Monasterio, I., Cakmak, I.: Biofortification strategies to increase grain zinc and iron concentrations in wheat. - J. Cereal Sci. 59: 365-372, 2014.

Vert, G., Barberon, M., Zelazny, E., Séguéla, M., Briat, J.F., Curie, C.: Arabidopsis IRT2 cooperates with the high-affinity 
iron uptake system to maintain iron homeostasis in root epidermal cells. - Planta 229: 1171-1179, 2009.

Vert, G., Briat, J.F., Curie, C.: Arabidopsis IRT2 gene encodes a root-periphery iron transporter. - Plant J. 26: 181-189, 2001.

Vert, G., Grotz, N., Dédaldéchamp, F., Gaymard, F., Guerinot, M.L., Briat, J.F., Curie, C.: IRT1, an Arabidopsis transporter essential for iron uptake from the soil and for plant growth. Plant Cell 14: 1223-1233, 2002.

Wang, L., Guo, K., Li, Y., Tu, Y., Hu, H., Wang, B., Cui, X., Peng, L.: Expression profiling and integrative analysis of the cesa/ csl superfamily in rice. - BMC Plant Biol. 10: 282, 2010.

White, P.J., Broadley, M.R., Hammond, J.P.: Bio-fortification of potato tubers using foliar zinc-fertiliser. - J. hort. Sci. Biotechnol. 87: 123-129, 2012.

White, P.J., Thompson, J.A., Wright, G.: Biofortifying Scottish potatoes with zinc. - Plant Soil 411: 151-165, 2017.

Wintz, H., Fox, T., Wu, Y.Y.: Expression profiles of Arabidopsis thaliana in mineral deficiencies reveal novel transporters involved in metal homeostasis. - J. biol. Chem. 278: 4764447653, 2003.
Yang, S., Zhang, X., Yue, J.X., Tian, D., Chen, J.Q.: Recent duplications dominate nbs-encoding gene expansion in two woody species. - Mol. Genet. Genomics 280: 187, 2008.

Yang, X., Huang, J., Jiang, Y.: Cloning and functional identification of two members of the ZIP (Zrt, Irt-like protein) gene family in rice (Oryza sativa L.). - Mol. Biol. Rep. 36: 281-287, 2009.

Yang, X.E., Chen, W.R., Feng, Y.: Improving human micronutrient nutrition through biofortification in the soil-plant system: China as a case study. - Environ. Geochem. Health 29: 413428, 2007.

Yusuf, G., McDonald, G.K., Graham, R.D.: Critical deficiency concentration of zinc in barley genotypes differing in zinc efficiency and its relation to growth responses. - J. Plant Nutr. 25: 545-560, 2002.

Zaman, Q.U., Aslam, Z., Yaseen, M.: Zinc biofortification in rice: leveraging agriculture to moderate hidden hunger in developing countries. - Arch. Agron. Soil Sci. 64: 147-161, 2017. 